\title{
Assistência de Enfermagem em Estratégias de Saúde da Família frente ao uso de substâncias psicoativas
}

\author{
Nursing Care in Family Health Strategies regarding psychoactive substance use
}

Atención de enfermería en estrategias de salud familiar con respecto al uso de sustancias psicoactivas

Ricardo Otávio Maia Gusmão, Rayssa Caroline de Oliveira ${ }^{\text {** }}$, Diego Dias de Araújo.

\section{RESUMO}

Objetivo: analisar a assistência dos enfermeiros em Estratégias Saúde da Família frente ao uso de substâncias psicoativas. Métodos: trata-se de um estudo quantitativo, transversal e descritivo com população constituída por 68 enfermeiros atuantes em Estratégias de Saúde da Família de um município do Norte de Minas Gerais, Brasil. Foi utilizado um questionário adaptado de Barros MA (2006). Os dados obtidos foram analisados por estatística descritiva com auxílio do software SPSS Statistics. O estudo foi aprovado por Comitê de Ética em Pesquisa. Resultados: verificou-se que $85 \%$ realizam o acolhimento; predomina o sentimento de insegurança $52,9 \%$ frente ao cuidado; $85,3 \%$ consideram que a maior dificuldade é a resistência dos usuários; $55,8 \%$ não realizam encaminhamentos aos serviços especializados e $70,5 \%$ relatam não receber contrareferência da Rede de Saúde Mental. Conclusão: é preciso investir na qualificação da assistência de enfermagem ofertada aos usuários de substâncias psicoativas. Os enfermeiros carecem de informações técnicas gerais que envolvam o uso, abuso e dependência de drogas. Acredita-se que uma assistência de enfermagem com qualidade possa garantir o tratamento adequado e favorecer a qualidade de vida dos usuários.

Palavras-chave: Assistência de enfermagem, Estratégia de saúde da família, Transtornos relacionados ao uso de substâncias.

\begin{abstract}
Objective: To analyze the assistance of nurses or Family Health Strategies regarding the use of psychoactive substances. Methods: This is a quantitative, cross-sectional and descriptive study with a population of 68 nurses working in Family Health Strategies in a city in northern Minas Gerais, Brazil. A questionnaire adapted from Barros MA (2006) was used. The obtained data were analyzed by descriptive statistics with the aid of the SPSS Statistics software. The study was approved by the Research Ethics Committee. Results: it was found that $85 \%$ perform the reception; the feeling of insecurity predominates $52.9 \%$ regarding care; $85.3 \%$ consider that the biggest difficulty is the resistance of users; $55.8 \%$ do not make referrals to specialized services and $70.5 \%$ report not receiving counter-referral from the Mental Health Network. Conclusion: it is necessary to invest in the qualification of nursing care offered to users of psychoactive substances. Nurses lack general technical information that involves drug use, abuse and dependence. It is believed that a quality nursing care can ensure the appropriate treatment and favor the quality of life of users.
\end{abstract}

Key words: Nursing care, Family health strategy, Substance use disorders.

\section{RESUMEN}

Objetivo: analizar la asistencia de enfermeras o estrategias de salud familiar en relación con el uso de sustancias psicoactivas. Métodos: Este es un estudio cuantitativo, transversal y descriptivo con una población de 68 enfermeras que trabajan en Estrategias de Salud Familiar de un municipio en el norte de Minas Gerais, Brasil. Se utilizó un cuestionario adaptado de Barros MA (2006). Los datos obtenidos se analizaron mediante

${ }^{1}$ Universidade Estadual de Montes Claros (UNIMONTES), Montes Claros-MG.

*E-mail: rayssacarololiveira@gmail.com

SUBMETIDO EM: 10/2019

ACEITO EM: 10/2019

PUBLICADO EM: 2/2020 
estadísticas descriptivas con la ayuda del software SPSS Statistics. El estudio fue aprobado por el Comité de Ética en Investigación. Resultados: se encontró que el $85 \%$ realiza la recepción; el sentimiento de inseguridad predomina en el $52.9 \%$ con respecto a la atención; El $85.3 \%$ considera que la mayor dificultad es la resistencia de los usuarios; El $55.8 \%$ no hace referencias a servicios especializados y el $70.5 \%$ informa que no recibe contra-referencias de la Red de Salud Mental. Conclusión: es necesario invertir en la calificación de los cuidados de enfermería ofrecidos a los usuarios de sustancias psicoactivas. Las enfermeras carecen de información técnica general que implique el uso de drogas, abuso y dependencia. Se cree que una atención de enfermería de calidad puede garantizar el tratamiento adecuado y favorecer la calidad de vida.

Palabras clave: Atención de enfermería, Estrategia de salud familiar, Trastornos por uso de sustancias.

\section{INTRODUÇÃO}

O terceiro Levantamento Nacional, realizado em 2015, sobre drogas no Brasil, evidenciou que 3,2 \% dos brasileiros fizeram uso de substâncias ilícitas nos 12 meses anteriores ao estudo. Isso equivale a $5 \%$ dos homens contra $1,5 \%$ entre as mulheres. Para a faixa etária de jovens de 18 a 24 anos, 7,4\% consumiram drogas ilícitas no mesmo período estimado (BRASIL, 2019).

A maconha revelou-se como a droga ilícita mais consumida no Brasil: 7,7\% dos brasileiros já usaram pelo menos uma vez na vida, considerando-se a faixa etária de 12 a 65 anos. Para esta mesma faixa etária, no entanto, os dados são mais preocupantes em relação ao consumo de álcool em que mais de $50 \%$ declararam ter feito uso da bebida em algum momento da vida. Considerando os últimos 30 dias, 30,1\% declararam ter feito uso de pelo menos uma dose e 2,3 milhões de pessoas apresentaram critérios para dependência de álcool (BRASIL, 2019).

Sobre o consumo de substâncias psicoativas, são várias as formas para se definir seu padrão de uso. 0 uso experimental de uma droga caracteriza-se por ser infrequente ou não persistente, em muitas situações se incluem como uso recreativo em circunstâncias sociais ou relaxantes; sem ainda ter implicações com dependência e outros problemas relacionados. O uso controlado referente à sustentação de um uso regular é aquele não compulsório e que não causa interferência nas atividades laborais do indivíduo. Já o uso nocivo/abusivo e a dependência são entendidos como um padrão de uso onde aumenta o risco de efeitos prejudiciais para o usuário. Porém, a dependência estabelece uma condição de comprometimento do usuário ao longo de um uso ininterrupto, relacionando-se com o grau de problemas causados pelas drogas (BRASIL, 2013).

O problema com uso de drogas é produto de um contexto político, cultural e socioeconômico que vem interferindo na escolha do sujeito, portanto deve ser entendido como um problema multidimensional e global, que não se restringe às relações entre o indivíduo e o consumo de drogas. Considerando que várias dimensões da vida do indivíduo são atingidas em função do uso/abuso de drogas (convívio social, relacionamento familiar, trabalho e saúde) e a abrangência do tipo de drogas que pode ser utilizada e seus efeitos adversos, verifica-se que as demandas por serviços de saúde pública também são diversificadas e abrangentes (TRIGO S, et al., 2015).

A atenção primária vem prestando assistência a milhões de brasileiros usuários de drogas garantindo-lhes acesso às ações e serviços de saúde e proporcionando a eles maior inclusão social, política e econômica a uma política sanitária (LIMA TL e ALVES ES, 2019). A Estratégia de Saúde da Família (ESF) trabalha dentro de uma lógica, com maior capacidade de ação para atender às necessidades de saúde da população de sua área de abrangência. A função da equipe de ESF é proporcionar uma assistência contínua à comunidade acompanhando integralmente a saúde da criança, do adulto, da mulher, dos idosos, a saúde mental, enfim, de toda a comunidade que vive no território sob sua responsabilidade (BRASIL, 2017).

A formação profissional de enfermagem em saúde mental e especificamente sobre a abordagem de álcool e drogas ainda é vista com muita dificuldade. Este pode ser um fator que implique em comprometimento da oferta de cuidados a esses usuários (PEREIRA AA, et al., 2015). 
De todos os profissionais da área da saúde, os enfermeiros atuam com maior proximidade com os usuários dos serviços, por isso eles possuem um maior potencial para identificar os problemas relacionados ao uso de drogas e desempenhar ações para assisti-los. Já no primeiro contato ele tem a capacidade de conhecer a história atual do consumo de drogas tais como: padrão de uso, problemas decorrentes do uso, realizar o acolhimento e sensibilizar-se com toda a situação provocada pelo uso de drogas. Além disso, o cuidado de enfermagem não está relacionado unicamente ao tratamento do usuário e de sua doença. Ele desempenha um papel de informação, educação preventiva e reinserção social do indivíduo, além de favorecer as ações de redução de danos (FARIAS LMS, et al.,2017).

Segundo o estudo realizado por Barros MA (2006), nota-se que as atitudes dos enfermeiros são neutras ou negativas frente aos problemas relacionados ao uso de drogas, na qual podem ser consequências de um ensino ainda precário. A formação de diferentes profissionais, de diferentes modos compromete a oferta de cuidados a esses usuários.

Percebendo as dificuldades que os profissionais enfermeiros apresentam para acolher e acompanhar os usuários de substâncias psicoativas, motivou-se investigar de que forma a assistência de enfermagem acontece nas ESFs de um município no Norte de Minas Gerais, Brasil. Sendo assim, a pesquisa possibilitará um conhecimento da realidade local referente à assistência de enfermagem na abordagem aos usuários de drogas.

Assim, a presente pesquisa teve como objetivo analisar a assistência dos enfermeiros em Estratégias Saúde da Família frente ao uso de substâncias psicoativas.

\section{MÉTODOS}

Trata-se de um estudo quantitativo, transversal, descritivo e analítico sobre ações dos profissionais enfermeiros das ESFs frente ao uso, abuso e dependência de drogas. A pesquisa quantitativa é aquela que adota estratégia sistemática, objetiva e rigorosa para gerar e apurar o conhecimento através da dedicação aos aspectos qualitativos da realidade (GIL AC, 2010).

A população de estudo foi constituída por 68 enfermeiros que atuam em ESFs de um município do Norte de Minas Gerais, Brasil. O referente município possui 136 enfermeiros de ESFs, sendo a amostra escolhida de forma aleatória e por conveniência. Utilizou-se na pesquisa um questionário adaptado do estudo denominado: Os profissionais do Programa Saúde da Família frente ao abuso e dependência de drogas, disponível em tese de mestrado da Universidade de São Paulo (BARROS MA, 2006).

A coleta de dados foi realizada em setembro e novembro de 2016. As análises descritivas foram feitas a partir dos dados coletados, compilados e tabulados pelo programa estatístico Statistical Package for the Social Sciences (SPSS) versão 23 sendo conduzida análise descritiva (frequência absoluta e percentual).

A pesquisa seguiu a portaria 466/12 do Ministério da Saúde para a realização de pesquisa com seres humanos. Os enfermeiros que integraram a amostra assinaram o Termo de Consentimento Livre e Esclarecido para realização do estudo ficando garantidos a privacidade, confidencialidade e anonimato das informações coletadas. A pesquisa foi avaliada e aprovada pelo Comitê de Ética com número de parecer 382.879, sendo respeitados todos os preceitos éticos.

\section{RESULTADOS E DISCUSSÃO}

Sobre a caracterização dos enfermeiros das ESFs em relação à idade e sexo, a maioria $27(39,7 \%)$ tem entre 30 a 34 anos, seguindo pela categoria entre 25 a 29 anos, 19 (27,95\%). Quanto ao sexo há uma predominância de profissionais do sexo feminino, $55(80,9 \%)$ do total. Em relação à formação profissional, 42 $(61,8 \%)$ dos enfermeiros afirmaram não ser especialista em saúde da família e/ou saúde mental. Sobre o tempo de trabalho na ESF, predominou-se o tempo de 2 a 5 anos, $26(38,2 \%)$ precedido pelo tempo de 7 meses a 1 ano, $16(23,5 \%)$. 
A predominância de enfermeiros não especialistas atuando na assistência pode implicar em dificuldades e despreparo para atender os usuários de drogas. Considera-se que o tempo de trabalho encontrado, em sua maioria, foi razoavelmente bom, inferindo experiência clínica. No entanto, é imprescindível que os enfermeiros das ESFs tenham conhecimentos básicos de saúde mental, a fim de garantir a integralidade do cuidado e o acolhimento adequado destes usuários.

Sobre as ações realizadas na prestação da assistência pelos enfermeiros na ESF aos usuários de substâncias psicoativas, 58 (85\%) relataram realizar o acolhimento; 44 (64\%) visitas domiciliares; 35 (51,5\%) consultas de enfermagem; 25 (36,8\%) grupos operativos (educação em saúde); 53 (77,9\%) encaminhar para a consulta médica; $42(61,8 \%)$ encaminhar para o serviço especializado e $3(4,4 \%)$ realizar outras ações.

Estes dados demonstram que os enfermeiros realizam muitas ações aos usuários de substâncias psicoativas na ESF. O acolhimento foi a atividade predominantemente mais realizada, mas chama a atenção que a prática de encaminhamento para consulta médica é mais comum que a realização da consulta de enfermagem a estes usuários.

De acordo com Brasil (2011), os profissionais do dispositivo da Atenção Básica e dos demais serviços da Rede de Atenção Psicossocial (RAPS) devem desenvolver ações que envolvem a proteção, promoção, prevenção, tratamento, reabilitação e redução de danos aos usuários de substâncias psicoativas.

Além disso, compete à assistência prestada pela equipe da ESF: escutar o paciente e ajudá-los em seus problemas emocionais; acompanhá-lo, buscando e estimulando-o a pensar as razões desse problema, como enfrentá-lo, promoção de atividades coletivas e intersetoriais, identificação de novos casos. Sendo que, quando o grau de complexidade ultrapassar as possibilidades dos profissionais não especializados, esses pacientes devem ser encaminhados para os serviços especializados da RAPS (SILVA PO, et al.,2018).

A descrição da assistência de saúde aos usuários de drogas das ESFs será apresentada, a seguir, tendo sido realizada por meio de porcentagem utilizando-se uma escala de 0 a 10 pontos, segundo observado na Tabela 1.

Conforme os enfermeiros, $46(67,6 \%)$ responderam a escala de 5 a abaixo, não ajudando ou ajudando pouco os pacientes com problemas relacionados ao abuso e dependência de drogas. Os dados são semelhantes ao estudo de Barros MA (2006), demonstrando que $64 \%$ dos profissionais da ESF percebem que pouco tem ajudado estes pacientes.

Tabela 1 - A apresentação de quanto os profissionais das ESFs percebe que tem ajudado os pacientes com problemas relacionados ao abuso e dependência de drogas, Montes Claros - MG $(n=68)$.

\begin{tabular}{cccc}
\multicolumn{2}{c}{ Escala de $\mathbf{0}$ a $\mathbf{1 0}$} & Número & Porcentagem \\
\hline Não Ajuda Nada & 0 & 2 & $2,9 \%$ \\
& 1 & 0 & $0 \%$ \\
2 & 5 & $7,4 \%$ \\
& 4 & 6 & $8,8 \%$ \\
& 5 & 10 & $14,7 \%$ \\
& 6 & 23 & $33,8 \%$ \\
& 7 & 6 & $8,8 \%$ \\
& 8 & 5 & $7,4 \%$ \\
& 9 & 6 & $8,8 \%$ \\
& 10 & 4 & $5,9 \%$ \\
& Total & 1 & $1,5 \%$ \\
\hline
\end{tabular}

Fonte: Gusmão ROM, et al., 2019. 
Analisando estes dados, percebemos que o enfermeiro tem feito pouco em relação ao cuidado prestado aos usuários de substâncias psicoativas no que diz respeito às atribuições requeridas para atuação na ESFs. Para Farias LMS, et al. (2017) é necessário repensar em como é oferecida a assistência a estes usuários, procurando meios de se desenvolver ações enfocadas na promoção, prevenção e recuperação dos agravos que os acometem. Percebe-se, em relação à enfermagem certa dificuldade na realização de suas práticas em saúde mental, ou seja, em desenvolver ações mais efetivas junto à comunidade.

O enfermeiro desempenha uma função primordial na ESFS, mas por outro lado, existe uma falta de interesse na saúde pública em desenvolver programas direcionados a esta clientela, assim como privilegiar capacitações nesta área, com ênfase nos riscos de problemas relacionados ao uso das drogas, as questões psicológicas envolvidas e a abordagem de ações educativas e que visem a prevenção deste agravo à saúde ou sua reabilitação (VARELA DSS, et al., 2015).

A Tabela 2 demonstrou que 36 (53\%) dos enfermeiros percebem o abuso/dependência de drogas como condições presentes entre os agravos à saúde na ESF, pontuando notas de 6 acima.

Tabela 2 - Apresentação de quanto os enfermeiros da ESF percebem que os problemas do abuso /dependência de drogas estão presentes entre os agravos à saúde na ESF, Montes Claros-MG $(n=6)$.

\begin{tabular}{cccc}
\hline \multicolumn{2}{c}{ Escala de $\mathbf{0}$ a $\mathbf{1 0}$} & Número & Porcentagem \\
\hline Não Muito & 0 & 4 & $5,9 \%$ \\
& 1 & 1 & $1,5 \%$ \\
2 & 2 & $2,9 \%$ \\
& 3 & 7 & $10,3 \%$ \\
4 & 6 & $8,8 \%$ \\
5 & 12 & $17,6 \%$ \\
6 & 7 & $10,3 \%$ \\
7 & 7 & $10,3 \%$ \\
8 & 8 & $11,8 \%$ \\
9 & 10 & 9 & $13,2 \%$ \\
& Total & 5 & $7,4 \%$ \\
\hline
\end{tabular}

Fonte: Gusmão ROM, et al., 2019.

A visão dos enfermeiros deste estudo corrobora com Lima PM, et al. (2014) que ressaltam, na atenção primária à saúde, um crescimento na demanda por parte dos usuários de substâncias psicoativas. Sendo assim, por conta dos frequentes agravos decorrentes do uso de substâncias, é necessário discutir sobre o cuidado desses usuários. Vale destacar que se considerando o princípio da Universalidade, os usuários de drogas e suas famílias possuem direitos que precisam ser garantidos nos diversos níveis de atenção à saúde.

Em outro estudo, Barros MA e Pillon (2007) reafirmam a ideia dos problemas decorrentes do uso e abuso de drogas estarem entre os problemas comuns na Atenção Primária. Assim, 61\% dos entrevistados relacionam os problemas referentes ao uso de drogas como situações frequentes que demandam cuidados. Já $89 \%$ referem ser muito importante cuidar desses usuários. No entanto, $69 \%$ referem dificuldades na assistência e que os usuários pouco se beneficiam da assistência oferecida. Para $64 \%$ dos entrevistados, a ESF pouco tem ajudado. 
Na Tabela 3, destaca-se que $44(64,7 \%)$ dos enfermeiros consideram como muito importante à assistência aos usuários de drogas inseridos na ESF.

Tabela 3 - Caracterização de quanto os enfermeiros das ESFs consideram importante a assistência aos usuários de drogas inserida na ESF, Montes Claros-MG $(n=68)$.

\begin{tabular}{|c|c|c|c|}
\hline \multicolumn{2}{|c|}{ Escala de 0 a 10} & \multirow{2}{*}{$\begin{array}{c}\text { Número } \\
0\end{array}$} & \multirow{2}{*}{$\begin{array}{c}\text { Porcentagem } \\
0 \%\end{array}$} \\
\hline & 0 & & \\
\hline & 1 & 0 & $0 \%$ \\
\hline & 2 & 0 & $0 \%$ \\
\hline & 3 & 0 & $0 \%$ \\
\hline & 4 & 0 & $0 \%$ \\
\hline & 5 & 2 & $2,9 \%$ \\
\hline & 6 & 1 & $1,5 \%$ \\
\hline & 7 & 3 & $4,4 \%$ \\
\hline & 8 & 5 & $7,4 \%$ \\
\hline & 9 & 13 & $19,1 \%$ \\
\hline Muito importante & 10 & 44 & $64,7 \%$ \\
\hline & & 68 & $100 \%$ \\
\hline
\end{tabular}

Fonte: Gusmão ROM, et al., 2019.

Tal dado é corroborado em estudo de Farias LMS, et al. (2017), na qual a maioria dos enfermeiros consideram muito importante assistir esses pacientes.

Além disso, os profissionais da atenção primária, convivem com a comunidade em que atuam, podendo desencadear mudanças expressivas na sua área de abrangência e zonas de influência, observando o cotidiano dessas pessoas (ROSENSTOCK KIV e NEVES MJ, 2010).

Em relação aos sentimentos dos enfermeiros ao lidarem com pacientes com problemas relacionados ao abuso/dependência de drogas, $36(52,9 \%)$ apresentam insegurança; precedidos por $25(36,8 \%)$ que apresentam receio (Tabela 4).

Em estudo realizado por Subrinho LQ, et al. (2018) apesar de os enfermeiros reconhecerem a necessidade de prestar um cuidado integral aos consumidores de drogas, revelam a sensação de despreparo e vislumbram a necessidade de capacitação. Sendo assim, inferimos que os sentimentos de insegurança e receio tem relação direta com essas sensações.

Para Kanno NP, et al. (2012), o cuidado por acontecer no território dos sujeitos os aproxima de situações como o tráfico de drogas. Assim, muitos se sentem expostos a situações de risco. Há também o temor de envolvimento excessivo com os usuários de drogas. 
Tabela 4 - Exposição dos sentimentos ao lidarem com pacientes com problemas relacionados ao abuso/dependência de drogas, pelos enfermeiros da ESF, Montes Claros- MG ( $n=68)$.

\begin{tabular}{|c|c|c|}
\hline Sentimento & Número & Porcentagem \\
\hline Bem Estar & 20 & $29,4 \%$ \\
\hline Mal Estar & 7 & $10,3 \%$ \\
\hline Desconforto & 21 & $30,9 \%$ \\
\hline Conforto & 7 & $10,3 \%$ \\
\hline Compaixão & 21 & $30,9 \%$ \\
\hline Confusão & 3 & $4,4 \%$ \\
\hline Estresse & 8 & $11,8 \%$ \\
\hline Raiva & 1 & $1,5 \%$ \\
\hline Indiferença & 3 & $4,4 \%$ \\
\hline Segurança & 7 & $10,3 \%$ \\
\hline Insegurança & 36 & $52,9 \%$ \\
\hline Contentamento & 5 & $7,4 \%$ \\
\hline Tristeza & 20 & $29,4 \%$ \\
\hline Alegria & 2 & $2,9 \%$ \\
\hline Insatisfação & 4 & $5,9 \%$ \\
\hline Satisfação & 19 & $27,9 \%$ \\
\hline Aceitação & 20 & $29,4 \%$ \\
\hline Receio & 25 & $36,8 \%$ \\
\hline Nervosismo & 5 & $7,4 \%$ \\
\hline Tédio & 0 & $0 \%$ \\
\hline Recusa & 1 & $1,5 \%$ \\
\hline Vergonha & 0 & $0 \%$ \\
\hline Curiosidade & 18 & $26,5 \%$ \\
\hline Medo & 18 & $26,5 \%$ \\
\hline Ansiedade & 19 & $27,9 \%$ \\
\hline Tranquilidade & 11 & $16,2 \%$ \\
\hline
\end{tabular}

Fonte: Gusmão ROM, et al., 2019.

A Tabela 5 expõe as dificuldades consideradas pelos enfermeiros na assistência aos usuários de drogas, sendo que a dificuldade mais considerada foi à resistência do usuário a assistência com 58 (85,3\%), seguida falta de capacitação profissional 56 (82,4\%). 
Tabela 5 - Apresentação das dificuldades considerada na assistência aos usuários de drogas, Montes ClarosMG $(n=68)$.

\begin{tabular}{lcc}
\multicolumn{1}{c}{ Dificuldades consideradas } & Número & Porcentagem \\
\hline Falta de capacitação profissional & 56 & $82,4 \%$ \\
Falta de articulação da rede & 41 & $60,3 \%$ \\
Deficiência de recursos & 48 & $70,6 \%$ \\
Resistência do usuário a assistência & 58 & $85,3 \%$ \\
Falta de colaboração familiar ao tratamento & 31 & $45,6 \%$ \\
Insegurança (risco de violência) & 40 & $58,8 \%$ \\
Outros & 2 & $2,9 \%$ \\
\hline
\end{tabular}

Fonte: Gusmão ROM, et al., 2019.

Para Farias LMS, et al. (2017) a falta de capacitação dos enfermeiros pode ser um fator desencadeante para o aumento na dificuldade de atuação com o usuário de drogas. Pressupõe-se assim que a falta conhecimento prático para desenvolvimento de ações inovadoras neste campo da drogadição dificulta 0 estabelecimento de vínculo e a busca ativa efetiva desse usuário.

Para os autores o ensino na Enfermagem sobre o uso e abuso de drogas não parece corresponder às demandas encontradas nos serviços de saúde. Destacam ainda que a universidade deve oferecer durante a graduação condições para que o futuro profissional adquira as competências necessárias para lidar com os usuários de drogas. Portanto, é na sua formação que o enfermeiro deve ter o aporte teórico e prático, para que o futuramente atue de forma adequada.

Ainda, para Subrinho LQ, et al. (2018) também há a identificação de problemas na capacitação e formação profissional, falta de envolvimento com o tema, restrição de tempo, sensação de ineficácia diante das dificuldades vivenciadas e insegurança ao envolvimento com os usuários de drogas.

O estudo evidenciou, a partir da apresentação sobre o ponto de vista e/ou preconceito dos enfermeiros à assistência aos usuários de substâncias psicoativas que $82,4 \%$ discordam que o uso ocasional de drogas não faz mal à saúde. Para $54,4 \%$, os dependentes de drogas usam drogas por problemas sociais e psicológicos e ele discorda na preferência em trabalhar com estes usuários. Já para $64,7 \%$ há discordância em referência ao fato de que o uso moderado de bebida alcoólica não seja prejudicial à saúde, enquanto $60,3 \%$ considera que mesmo cuidando do usuário de substâncias, ele tende a continuar consumindo a substância, mesmo em tratamento (Tabela 6).

O estudo realizado Subrinho LQ, et al. (2018) demonstrou que os enfermeiros reconhecerem a necessidade de prestar um cuidado integral aos consumidores de drogas, no entanto eles desenvolvem uma assistência com foco prioritário a abstinência. Ainda, culpabilizam esses consumidores pelo insucesso no tratamento, ao considerá-los não responsivos ao cuidado, ao mesmo tempo em que desvelam a sensação de despreparo para atender esta demanda.

Compreendemos que a estratégia de Redução de Danos consiste em uma nova perspectiva de cuidado com o consumidor de drogas, e o seu foco não é na abstinência. 
Tabela 6 - Apresentação do ponto de vista e/ou preconceito dos enfermeiros ao lidarem com usuários de drogas, Montes Claros-MG ( $n=68)$.

\begin{tabular}{|c|c|c|c|c|c|c|}
\hline \multirow{2}{*}{ Considera } & \multicolumn{2}{|c|}{ Concorda } & \multicolumn{2}{|c|}{ Indiferente } & \multicolumn{2}{|c|}{ Discordo } \\
\hline & Número & $\%$ & Número & $\%$ & Número & $\%$ \\
\hline $\begin{array}{l}\text { Dependentes de drogas não estão } \\
\text { preocupados com seu estilo de vida }\end{array}$ & 28 & $41,20 \%$ & 14 & $20,60 \%$ & 26 & $\begin{array}{c}38,20 \\
\%\end{array}$ \\
\hline $\begin{array}{l}\text { Drogas usadas ocasionalmente não fazem } \\
\text { mal à saúde }\end{array}$ & 10 & $14,70 \%$ & 19 & $2,90 \%$ & 56 & $\begin{array}{c}82,40 \\
\%\end{array}$ \\
\hline $\begin{array}{l}\text { Dependentes de drogas são mais } \\
\text { susceptíveis às doenças físicas }\end{array}$ & 57 & $83,80 \%$ & 19 & $2,90 \%$ & 8 & $\begin{array}{c}11,80 \\
\%\end{array}$ \\
\hline $\begin{array}{l}\text { Prefiro trabalhar com dependentes de } \\
\text { drogas a outro tipo de paciente }\end{array}$ & 4 & $5,90 \%$ & 27 & $39,70 \%$ & 37 & $\begin{array}{c}54,40 \\
\%\end{array}$ \\
\hline $\begin{array}{l}\text { Os dependentes de drogas respeitam seus } \\
\text { familiares }\end{array}$ & 1 & $1,50 \%$ & 18 & $26,50 \%$ & 49 & $\begin{array}{c}72,10 \\
\%\end{array}$ \\
\hline $\begin{array}{l}\text { Os dependentes de drogas merecem um } \\
\text { espaço no hospital, como qualquer outro } \\
\text { paciente }\end{array}$ & 56 & $82,40 \%$ & 4 & $5,90 \%$ & 8 & $\begin{array}{c}11,80 \\
\%\end{array}$ \\
\hline $\begin{array}{l}\text { Os dependentes de drogas querem parar de } \\
\text { usar tais substâncias }\end{array}$ & 15 & $22,10 \%$ & 35 & $51,50 \%$ & 18 & $\begin{array}{c}26,50 \\
\%\end{array}$ \\
\hline $\begin{array}{l}\text { Os dependentes de drogas usam drogas por } \\
\text { problemas sociais e psicológicos }\end{array}$ & 37 & $54,40 \%$ & 19 & $27,90 \%$ & 12 & $\begin{array}{c}17,60 \\
\%\end{array}$ \\
\hline $\begin{array}{l}\text { Todos os dependentes de drogas precisam } \\
\text { de consultas psiquiátricas }\end{array}$ & 36 & $52,90 \%$ & 12 & $17,60 \%$ & 20 & $\begin{array}{c}29,40 \\
\%\end{array}$ \\
\hline $\begin{array}{l}\text { Eu me sinto bem quando trabalho com } \\
\text { dependentes de drogas. }\end{array}$ & 18 & $26,50 \%$ & 27 & $39,70 \%$ & 23 & $\begin{array}{c}33,80 \\
\%\end{array}$ \\
\hline $\begin{array}{l}\text { A maioria dos dependentes de drogas não } \\
\text { gosta de ser dependentes }\end{array}$ & 36 & $52,90 \%$ & 25 & $36,80 \%$ & 7 & $\begin{array}{c}10,30 \\
\%\end{array}$ \\
\hline $\begin{array}{l}\text { Os dependentes de drogas são pessoas } \\
\text { isoladas e solitárias. }\end{array}$ & 24 & $35,30 \%$ & 17 & $25,00 \%$ & 27 & $\begin{array}{c}39,70 \\
\%\end{array}$ \\
\hline $\begin{array}{l}\text { Quando usada moderadamente, a bebida } \\
\text { alcoólica não é prejudicial à saúde }\end{array}$ & 19 & $27,90 \%$ & 5 & $7,40 \%$ & 44 & $\begin{array}{c}64,70 \\
\%\end{array}$ \\
\hline A dependência de drogas é uma doença & 58 & $85,30 \%$ & 9 & $13,20 \%$ & 1 & $1,50 \%$ \\
\hline $\begin{array}{l}\text { Embora eu possa ajudar o dependente de } \\
\text { drogas, ele ainda pode continuar usando, } \\
\text { mesmo em tratamento }\end{array}$ & 41 & $60,30 \%$ & 8 & $11,80 \%$ & 19 & $\begin{array}{c}27,90 \\
\%\end{array}$ \\
\hline
\end{tabular}

Fonte: Gusmão ROM, et al., 2019.

A Associação Internacional de Redução de Danos a define como: um conjunto de políticas e práticas cujo objetivo é reduzir os danos associados ao uso de drogas psicoativas em pessoas que não podem ou não querem parar de usar drogas. Por definição, redução de danos foca na prevenção aos danos, ao invés da prevenção do uso de drogas; bem como foca em pessoas que seguem usando drogas. Refere-se a políticas, 
programas e práticas que visam primeiramente reduzir as consequências adversas para a saúde, sociais e econômicas do uso de drogas lícitas e ilícitas, sem necessariamente reduzir o seu consumo. Redução de Danos beneficia pessoas que usam drogas, suas famílias e a comunidade (RIO DE JANEIRO, 2016).

Evidentemente há uma recusa a priori da equipe em atender estes pacientes e ainda, por apresentarem dificuldades concretas em lidar com eles. Os profissionais de saúde sentem-se incomodados diante da demanda dessa clientela sem cura, desafiando os seus saberes, e provocando uma sensação de impotência (RIO DE JANEIRO, 2016). O estudo ainda conseguiu demonstrar que 38 (55,8\%) dos enfermeiros não conseguem identificar os casos que devem ser encaminhados aos serviços especializados. Quanto ao receber à contrarreferência feita pelos serviços especializados, 48 (70,5\%) declararam que não recebem, 0 que também compromete o cuidado destes usuários.

As diretrizes do Ministério da Saúde recomendam que a assistência em saúde mental se amplie em diversos serviços da Rede de Atenção Psicossocial como a ESF, consultório na rua, Centros de Atenção Psicossocial (CAPS), ambulatórios e hospitais gerais, tornando uma rede bem articulada, para que ocorra uma maior inclusão social dos usuários. Entretanto, percebe-se que há dificuldades nestes processos de integração. Assim, os inacabáveis encaminhamentos determinam a desarticulação das condutas e torna fragmentada a relação entre os serviços levando a inviabilidade do atendimento de ausência de um cuidado integral e transdisciplinar (MACEDO JP, et al., 2017).

\section{CONCLUSÃO}

Conclui-se que a assistência de enfermagem ofertada aos usuários de drogas precisa ser aprimorada. Os enfermeiros apresentam dificuldades consideráveis na assistência oferecida que pode ser decorrente da falta de conhecimentos. Apesar dos enfermeiros das ESFs considerarem que os problemas relacionados às drogas estão inseridos entre os agravos à saúde e a importância de assistir esses pacientes, apresentam também sentimentos negativos, preconceito e focam o cuidado na abstinência. Neste contexto, considera-se necessário investir no desenvolvimento de competências e formação do enfermeiro, a fim de favorecer a melhoria da assistência de enfermagem e contribuição para a qualidade de vida desses usuários.

\section{REFERÊNCIAS}

1. BARROS MA, PILLON SC. Assistência aos usuários de drogas: a visão dos profissionais do programa saúde da família. Revista Enfermagem UERJ, 2007; 15(2): 261-266.

2. BARROS MA. Os profissionais do Programa Saúde da Família frente ao uso, abuso e dependência de drogas. RP. Dissertação (Mestrado em Enfermagem Psiquiátrica) - Escola de Enfermagem de Ribeirão Preto, Universidade de São Paulo, São Paulo. 2006; 150p.

3. BRASIL. Fundação Oswaldo Cruz. Pesquisa revela dados sobre o consumo de drogas no Brasil. Rio de Janeiro, 2019.

4. BRASIL. Ministério da Saúde. Biblioteca Virtual em Saúde. A política do ministério da saúde para a atenção integral a usuários de álcool e outras drogas. Brasília, 2004.

5. BRASIL. Ministério da Saúde. Portaria no. 2.436 de 21 de setembro de 2017. Aprova a Política Nacional de Atenção Básica, estabelecendo a revisão de diretrizes para a organização da Atenção Básica, no âmbito do Sistema Único de Saúde (SUS). Brasília, 2017.

6. FARIAS LMS, et al. O enfermeiro e a assistência a usuários de drogas em serviços de atenção básica. Revista de Enfermagem UFPE, 2017; 11:2871-2880.

7. GIL C. Como Elaborar Projetos de Pesquisa. 5 ed. São Paulo: Atlas, 2010.

8. KANNO NP, et al. Profissionais da Estratégia Saúde da Família diante de demandas médico-sociais: dificuldades e estratégias de enfrentamento. Saúde e Sociedade, 2012; 21(4): 884-894.

9. LIMA PM, et al. Assistência ao usuário de drogas na atenção primária à saúde. Psicologia em Estudo, 2014, 19(2): 223-233.

10. LIMA TL, ALVES ES. Reflexões sobre o cuidado com as pessoas que fazem uso abusivo de drogas na atenção básica. Humanae. Questões controversas do mundo contemporâneo, 2019; 13(2) 1-19 
11. MACEDO JP, et.al. A regionalização da atenção psicossocial em álcool e outras drogas no Brasil. Tempus Actas de Saúde coletiva, 2017; 11(3): 144-162.

12. PEREIRA AA, et al. Formação dos enfermeiros em saúde mental que atuam na atenção primária à saúde: contribuições teóricas. Sanare, Revista de Políticas Públicas, 2015; 14(1):08-14.

13. RIO DE JANEIRO. Secretaria Municipal de Saúde. Coleção Guia de Referência Rápida Álcool e outras drogas: tratamento e acompanhamento de pessoas com problemas relacionados ao uso de álcool e outras drogas. 1 ed. Rio de Janeiro, 2016.

14. ROSENSTOCK KIV, NEVES MJ. Papel do enfermeiro da atenção básica de saúde na abordagem ao dependente de drogas em João Pessoa. Revista Brasileira de Enfermagem, 2010; 63 (4): 581-586.

15. SILVA PO, et al. Cuidado clínico de enfermagem em saúde mental. Rev enferm UFPE online. 2018;12(11):3133-46.

16. SUBRINHO LQ, et al. Cuidado ao consumidor de drogas: percepção de enfermeiros da Estratégia de Saúde da Família. Saúde e Sociedade, 2018; 27(3): 834-844.

17. TRIGO S, et al. Representações sociais de adolescentes sobre o consumo de drogas. Arquivos de Medicina. 2015; 29(2): 39-45.

18. VARELA DSS, et al. Dificuldades de enfermeiros no trabalho com usuários de álcool e outras drogas: revisão integrativa. Revista de Enfermagem UFPE, 2015; 9(10): 9576-9583. 